\title{
A Statistical Model for Angle of Arrival in Indoor Multipath Propagation
}

\author{
Quentin Spencer, Michael Rice, Brian Jeffs, and Michael Jensen \\ Department of Electrical \& Computer Engineering \\ Brigham Young University \\ Provo, Utah 84602
}

\begin{abstract}
Multiple antenna systems are a useful way of overcoming the effects of multipath interference, and can allow more efficient use of spectrum. In order to test the effectiveness of various algorithms such as diversity combining, phased array processing, and adaptive array processing in an indoor environment, a channel model is needed which models both the time and angle of arrival in indoor environments. Some data has been collected indoors and some temporal models have been proposed, but no existing model accounts for both time and angle of arrival. This paper discusses existing models for the time of arrival, experimental data that were collected indoors, and a proposed extension of the Saleh-Valenzuela model [1], which accounts for the angle of arrival. Model parameters measured in two different buildings are compared with the parameters presented in the paper by Saleh and Valenzuela, and some statistical validation of the model is presented.
\end{abstract}

\section{INTRODUCTION}

There have been many different approaches for overcoming the problem of multipath interference, both in outdoor and indoor applications. Some of them include channel equalization, directional antennas, and multiple antenna systems, each being more particularly suited to different applications. The use of multiple antenna systems can be particularly useful for indoor applications such as local area networks, because they allow the possibility of communicating with multiple users simultaneously over a single frequency band, increasing throughput and making efficient use of frequency spectrum. The signals from different antennas can be combined in various ways, including diversity combining, phased array processing, and adaptive array algorithms. Adaptive array sytems are becoming increasingly feasible for high bandwidth applications with continuing improvements in digital signal processors. In addition, the availability of new, higher frequency bands has made wireless networks an increasinly attractive and feasible option. The effects of multipath interference have been studied extensively in various outdoor scenarios. However, the study of the indoor multipath channel is relatively new. In order to be able to predict the performance of indoor communications systems, models are needed that accurately model the behavior of radio transmissions in indoor environments.

Several other researchers have already collected various types of data on indoor mulipath propagation. The foundation for much of today's work was by Turin, et al [2], which was a study of outdoor multipath propagation in an urban environment. The first model for indoor multipath propagation was proposed by Saleh and Valenzuela
[1], whose work was based on the work of Turin. Their work consisted of collecting temporal data on indoor propagation, from which they proposed a time domain model for indoor propagation.

Most indoor propagation research has dealt with the time of arrival and paid little attention to the angle of arrival. In order to predict the performance of adaptive array systems, the angle of arrival is very important information. Some recent papers have begun to address the angle of arrival. Lo and Litva [3] found that multipath arrivals tend to occur at varying angles indoors, but were not able to arrive at any conclusions based on their limited data. Guerin [4] collected angular and temporal data separately, but did not correlate the two. Wang, et al [5], used a rectangular array to estimate both the elevation and azimuth angles of arrival for major multipaths, but did not measure the corresponding time of arrival. Litva, et al, [6] collected simultaneous time and angle of arrival data, similar to the format of the data used in this paper. They came to the preliminary conclusion that it is possible to make accurate measurements of this type and learn more about what is happening in the indoor multipath channel. However, their experiment was not extensive enough to make any conclusions about the channel.

This paper presents an extension to the Saleh-Valenzuela model which accounts for the angle of arrival. This is based on data that includes information about both the time and angle of arrival, presented in [7]. The Saleh-Valenzuela model is explained, and the new data is discussed. Model parameters based on the new data are derived and compared to the parameters found by Saleh and Valenzuela at a lower frequency.

\section{The Saleh-Valenzuela Model}

The model proposed by Saleh and Valenzuela is based on a clustering phenomenon observed in their experimental data. In all of their observations, the arrivals came in one or two large groups within a 200 ns observation window. It was observed that the second clusters were attenuated in amplitude, and that rays, or arrivals within a single cluster, also decayed with time. Their model proposes that both of these decaying patterns are exponential with time, and are controlled by two time constants: $\Gamma$, the cluster arrival decay time constant, and $\gamma$, the ray arrival decay time constant. Fig. 1 illustrates this, showing the mean envelope of a three cluster channel. 
The impulse response of the channel is given by:

$$
h(t)=\sum_{l=0}^{\infty} \sum_{k=0}^{\infty} \beta_{k l} \delta\left(t-T_{l}-\tau_{k l}\right),
$$

where the sum over $l$ represents the clusters, and the sum over $k$ represents the arrivals within each cluster. The amplitude of each arrival is given by $\beta_{k l}$, which is a Rayleigh distributed random variable, whose mean square value is described by the double-exponential decay illustrated in Fig. 1. Mathematically it is given by:

$$
\begin{aligned}
\overline{\beta_{k l}^{2}} & =\overline{\beta^{2}\left(T_{l}, \tau_{k l}\right)} \\
& =\overline{\beta^{2}(0,0)} e^{-T_{l} / \Gamma} e^{-\tau_{k l} / \gamma},
\end{aligned}
$$

where $\overline{\beta^{2}(0,0)}$ is the average power of the first arrival of the first cluster. This average power is determined by the separation distance of transmitter and receiver.

The time of arrival is described by two Poisson processes which model the arrival times of clusters and the arrival times of rays within clusters. The time of arrival of each cluster is an exponentially distributed random variable conditioned on the time of arrival of the previous cluster. The case is the same for each ray, or arrival within a cluster. Following the terminology used by Saleh and Valenzuela, rays shall refer to arrivals within clusters, so that the cluster arrival rate implies the parameter for the intercluster arrival times and the ray arrival rate refers to the parameter for the intracluster arrival times. The distributions of these arrival times are shown in equations 4 and 5:

$$
\begin{aligned}
p\left(T_{l} \mid T_{l-1}\right) & =\Lambda e^{-\Lambda\left(T_{l}-T_{l-1}\right)} \\
p\left(\tau_{k l} \mid \tau_{(k-1) l}\right) & =\lambda e^{-\lambda\left(\tau_{k l}-\tau_{(k-1) l}\right)}
\end{aligned}
$$

where $\Lambda$ is the cluster arrival rate, and $\lambda$ is the ray arrival rate. In their data, Saleh and Valenzuela did not have any information on angle of arrival, and assumed that the angles of arrival were uniformly distributed over the interval $[0,2 \pi)$.

Other indoor multipath models have been proposed, such as the model proposed by Ganesh and Pahlavan [8], but they will not be discussed here. The data used in this paper fit the Saleh-Valenzuela model well, and as a result the model was chosen as the basis for the extended model presented here.

\section{Experimental Data}

In order to analyze and model the indoor multipath channel, a data gathering apparatus was designed which was able to take simultaneous measurements of the time and angle of arrival. The frequency band was from 6.75 to $7.25 \mathrm{GHz}$. Using the system, a total of 65 data sets were collected in two buildings on the Brigham Young University Campus. In the Clydo building, a reinforced concrete and cinder block building, 55 data sets were collected. For comparison, ten additional data sets were collected in the
Crabtree Building, constructed mostly of steel and gypsum board. Each data set can be viewed as an image plot, with angle as one axis, and time as the second axis. A typical data set is pictured in Fig. 2. The images were processed to remove blurring effects so that the precise time, angle and amplitude of each major multipath arrival is known. The data collection and processing is discussed in greater detail in [7].

Visual observation of the data showed that clustering like that observed by Saleh and Valenzuela was present in the data. The nature of the clustering tended to follow the model of Saleh and Valenzuela quite well. In general, the strength of clusters tended to decay with increasing delay times, and arrivals within each cluster showed a similar pattern of decay. One difference from the Saleh-Valenzuela data is the higher average number of clusters per data set.

\section{A Proposed Time/Angle Model for Indoor Multipath Propagation}

In this section we propose a statistical model for the indoor multipath channel that includes a modified version of the Saleh-Valenzuela model, and incorporates an angle-ofarrival model. In addition, methods of estimating parameters from the data are discussed.

\section{A. Time of Arrival}

The time and amplitude of arrival portion of the combined model is represented by $h(t)$ in equation (1), where, as before, $\overline{\beta_{k l}^{2}}$ is the mean square value of the $k$ th arrival of the $l$ th cluster. This mean square value is described by the exponential decay given in equation (3) and illustrated in Fig. 1.

As before, the ray arrival time within a cluster is given by the Poisson distribution of equation (5), and the first arrival of each cluster is given by $T_{l}$, described by the Poisson distribution of (4). The inter-ray arrival times, $\tau_{k l}$, are dependent on the time of the first arrival in the cluster $T_{l}$. In the Saleh-Valenzuela model, the first cluster time $T_{1}$ was dependent on $T_{0}$ which was assumed to be zero. With the estimated parameter in [1] of $1 / \Lambda \approx 300 \mathrm{~ns}$, the first arrival time will typically be in the range of 200 to 300 $\mathrm{ns}$, which is a reasonable figure. However, a problem with this was found when the $\Lambda$ parameter in the new data was discovered to be very low, but the delay time to the first arrival was often still on the order of $200 \mathrm{~ns}$. Under the Saleh-Valenzuela model, this would make any long delays which would occur at larger separation distances between transmitter and receiver highly improbable. To remedy this problem, it is proposed that $T_{0}$ be the line of sight propagation time:

$$
T_{0}=\frac{r}{c},
$$

where $c$ is the speed of light, and $r$ is the separation distance. This allows for the time of the first arrival to be more directly dependent on the separation distance. 


\section{B. Angle of Arrival}

It will be assumed that time and angle are statistically independent. If there were a correlation, it would be expected that a longer time delay would correspond to a larger angular variance from the mean of a cluster. This was not observed in the data, so at this point an assumption of independence is reasonable, but further study of the correlation structure may be warranted. The consequence of this independence is that the complete impulse response with respect to both time and angle, which we will call $h(t, \theta)$, becomes a separable function:

$$
h(t, \theta) \approx h(t) h(\theta) .
$$

As a result, $h(\theta)$ can be be addressed separately from $h(t)$.

We propose an independent angular impulse response of the system, similar to the time impulse response of the channel given in 1 :

$$
h(\theta)=\sum_{l=0}^{\infty} \sum_{k=0}^{\infty} \beta_{k l} \delta\left(\theta-\Theta_{l}-\omega_{k l}\right),
$$

where, as before, $\beta_{k l}$ is the ray amplitude for the $k$ th arrival in the $l$ th cluster, given in equations (2) and (3). $\Theta_{l}$ is the mean angle of each cluster, which is distributed uniformly on the interval $[0,2 \pi)$. We propose that the ray angle within a cluster, $\omega_{k l}$, be modeled as a zero mean Laplacian distribution with standard deviation $\sigma$ :

$$
p(\theta)=\frac{1}{\sqrt{2} \sigma} e^{-|\sqrt{2} \theta / \sigma|} .
$$

The correlation of these distributions to the data is shown in the next section.

\section{Parameter Estimation}

This section outlines methods of deriving the distributions and estimating the parameter $\sigma$ given in the previous section. The distribution parameters of the cluster means, $\Theta_{l}$, is found by identifying each of the clusters in a given data set. The mean angle of arrival for each cluster is calculated. In order to remove the specific room geometry and orientation, the first arrival (in time) for each data set is taken as the reference. The relative cluster means are calculated by subtracting the mean of the reference cluster from all other cluster means. To estimate the distribution of cluster means over the ensemble of all data sets, a histogram can be generated of all relative cluster means, disregarding the first clusters (since their relative mean is always 0 ).

The procedure to estimate $\sigma$ is similar. The cluster mean is subtracted from the absolute angle of each ray in the cluster to give a relative arrival angle with respect to the cluster mean. The relative arrivals are collected over the ensemble of all data sets, and a histogram can be generated. Using a least mean square algorithm, the histogram is fit to the closest Laplacian distribution, which gives the value for $\sigma$.

\section{Using the Model}

The extended model for $h(t, \theta)$ is useful for analysis or simulation of array processing algorithms that might be used in an indoor environment. In order, for example, to conduct a Monte Carlo simulation of an array antenna processor, it is necessary to generate a random channel using the statistical model. This section outlines the procedure for doing so.

The first step is to choose the transmitter/receiver separation distance $r$, which can be chosen either randomly or arbitrarily. Knowing $r$, the next step is to determine $\overline{\beta^{2}(0,0)}$, the mean power of the first arrival, which is given by

$$
\overline{\beta^{2}(0,0)} \approx \frac{G(1 m) r^{-\alpha}}{\gamma \lambda},
$$

where $G(1 \mathrm{~m})$ is the channel gain at $r=1$ meter, and $\alpha$ is a channel loss parameter. $\gamma$ and $\theta$ are respectively the ray decay parameter and ray arrival rate in the model for $h(t)$. Equation (10) is derived and the characteristics of $\alpha$ in the indoor environment are discussed in greater detail in $[1]$.

After $\overline{\beta^{2}(0,0)}$ is determined, the next step is to determine the cluster and ray arrival times. The corresponding distributions are given in equations (4) and (5), where $T_{0}=r / c$. After the times are determined, the mean amplitudes $\overline{\beta_{k l}}$ are determined by equation 3 . The actual amplitudes for each arrival, $\beta_{k l}$, are determined by sampling a Rayleigh distribution whose mean is $\overline{\beta_{k l}}$.

The angles are determined by first randomly choosing the cluster angles, which are uniformly distributed from 0 to $2 \pi$. Relative ray angles are then determined by sampling a Laplacian distribution as given in equation (9).

\section{Model Parameters from the Data}

The intercluster time decay constant, $\Gamma$, was estimated by normalizing the cluster amplitudes (the amplitude of the first arrival) so that the first one had an amplitude of 1 and a time delay of 0 . All of the cluster amplitudes were superimposed as shown in Fig. 3. The estimate for $\Gamma$ was found by curve fitting the line (representing an exponential curve) to minimize the mean squared error. The values for $\Gamma$ and $\gamma$ were estimated for both buildings in a similar manner. In this particular example, the fit is less than ideal, but it was better in the other cases, especially when there were more data points. In their data, Saleh and Valenzuela did not have exact amplitudes available, and as a result were not able to use curve fitting or generate plots as in Fig. 3. Their parameters were as a result very rough estimates, but they did observe the same general decay trend as in this data, which supports the exponential decay model.

The Poisson parameters, $\Lambda$ and $\lambda$, representing the intercluster and intracluster arrival rates were estimated by subtracting each arrival time from its predicessor to produce a set of conditional arrival times $p\left(\tau_{k l} \mid \tau_{(k-1) l}\right)$. The 


\begin{tabular}{lrrr} 
parameter & $\begin{array}{r}\text { Clyde } \\
\text { Building }\end{array}$ & $\begin{array}{r}\text { Crabtree } \\
\text { Building }\end{array}$ & $\begin{array}{r}\text { Saleh- } \\
\text { Valenzuela }\end{array}$ \\
\hline$\Gamma$ & $33.6 \mathrm{~ns}$ & $78.0 \mathrm{~ns}$ & $60 \mathrm{~ns}$ \\
$\gamma$ & $28.6 \mathrm{~ns}$ & $82.2 \mathrm{~ns}$ & $20 \mathrm{~ns}$ \\
$1 / \Lambda$ & $16.8 \mathrm{~ns}$ & $17.3 \mathrm{~ns}$ & $300 \mathrm{~ns}$ \\
$1 / \lambda$ & $5.1 \mathrm{~ns}$ & $6.6 \mathrm{~ns}$ & $5 \mathrm{~ns}$ \\
$\sigma$ & $25.5^{\circ}$ & $21.5^{\circ}$ & -
\end{tabular}

Table 1. A comparison of model parameters for the two buildings and from the Saleh-Valenzuela paper [1]

probability distribution of these with the best fitting pdf (for the Clyde Building) is shown in Fig. 4.

Fig. 5 shows a CDF of the relative cluster angles for the Clyde Building, illustrating the relatively uniform distribution of clusters in angle. The same was true in the Crabtree Building. The distribution of the ray arrivals with respect to the cluster mean is shown in Fig. 6. The sharp peak at the mean is characteristic of a Laplacian distribution. The superimposed curve is a Laplacian distribution that was fit by integrating a Laplacian PDF over each bin, and matching the curves using a least mean square goodness of fit measure. The Laplacian distribution turns out to be a very close fit in both buildings.

Table 1 shows a comparison of the model parameters estimated for the Clyde Building, the Crabtree Building, and those estimated by Saleh and Valenzuela from their data. The most obvious discrepancy is in the estimates for the value of $\Lambda$. This is due to the fact that there were significantly more clusters observed in both the Clyde and Crabtree buildings compared to an average of 1-2 clusters observed by Saleh and Valenzuela. This may be partly due to the higher RF frequency, but the more likely cause is the ability of our testbed to see clusters that were close together in time, but separated in angle. Another interesting phenomenon is that $\Gamma$ is very low in the Clyde Building, and $\gamma$ is larger than $\Gamma$ in the Crabtree Building, meaning that the Clyde Building tends to attenuate more than the Crabtree Building. The values of $\sigma$ were close in both buildings, and there is no precedent for comparison with other data.

\section{CONCLUSION}

Many aspects of the model have plausible physical explanations. Because an absolute angular reference was maintained during the collection of the data, it was possible to compare the processed data with the geometry of each configuration. The strongest cluster was almost always associated with the direct line of sight, even when there were walls blocking the line of sight path. Apparent causes of weaker clusters were back wall reflections and doorway openings. It is likely that each cluster corresponds to a major path to the receiver, and the arrivals within each cluster are likely the result of smaller, closely associated objects that are part of a very similar group of paths to the receiver. These paths will take slightly longer to ar- rive than the first arrival in the cluster, and are usually attenuated relative to this first arrival.

The amplitudes of clusters and rays within clusters both follow the same pattern of exponential decay over time observed by Saleh and Valenzuela. The differences in model parameters are likely due to the difference in frequency (Saleh and Valenzuela used $1.5 \mathrm{GHz}$ ). The other discrepancy is in the markedly faster cluster arrival rate, which is most likely explained by the larger overall number of clusters resulting from a more sensitive data gathering apparatus. The model parameters for the Clyde and Crabtree Buildings were in general very similar. The most notable exception is the extremely slow amplitude decay of rays within a cluster in the Crabtree building. In general, the model seemed to be able to accurately describe the differing multipath characteristics in both buildings, regardless of their very different construction. This implies that the model could possibly provide a general representation for many different types of buildings, and model parameters could therefore be found for other types of buildings.

The angle-of-arrival model presented here, though yet unconfirmed, is a strong alternative to only previous option for simulation: random assignment of angles or guessing at the anglular properties of the channel. The most important area for continued research is applying the model for its intended purpose-comparison of array processing algorithms. This can be done either by mathematical analysis or Monte Carlo simulation. A mathematical analysis is likely intractible due to the large number of variables in the model, but the model can be a very useful tool for the generation of random multipath channels for simulation.

\section{REFERENCES}

[1] Adel A. M. Saleh and Reinaldo A. Valenzuela. A statistical model for indoor multipath propagation. IEEE Journal on Selected Areas of Communications, SAC-5:128-13, February 1987.

[2] George L. Turin et al. A statistical model of urban multipath propagation. IEEE Transactions on Vehicular Technology, VT21(1):1-9, February 1972.

[3] T. Lo and J. Litva. Angles of arrival of indoor multipath. Electronics Letters, 28(18):1687-1689, August 271992.

[4] Stephane Guerin. Indoor wideband and narrowband propagation measurements around $60.5 \mathrm{ghz}$. in an empty and furnished room. In IEEE Vehicular Technology Conference, pages 160$164,1996$.

[5] Jian-Guo Wang, Ananda S. Mohan, and Tim A Aubrey. Anglesof-arrival of multipath signals in indoor environments. In IEEE Vehicular Technology Conference, pages 155-159. IEEE, 1996.

[6] John Litva, Amir Ghaforian, and Vytas Kezys. High-resolution measurements of aoa and time-delay for characterizing indoor propagation environments. In IEEE Antennas and Propagation Society International Symposium 1996 Digest, volume 2, pages 1490-1493. IEEE, 1996.

[7] Quentin Spencer, Michael Rice, Brian Jeffs, and Michael Jensen. Indoor wideband time/angle of arrival multipath propagation results. In IEEE Vehicular Technology Conference. IEEE, 1997.

[8] R. Ganesh and K. Pahlavan. Statistical modeling and computer simulation of indoor radio channel. IEE Proceedings-I, 138(3):153-161, June 1991. 


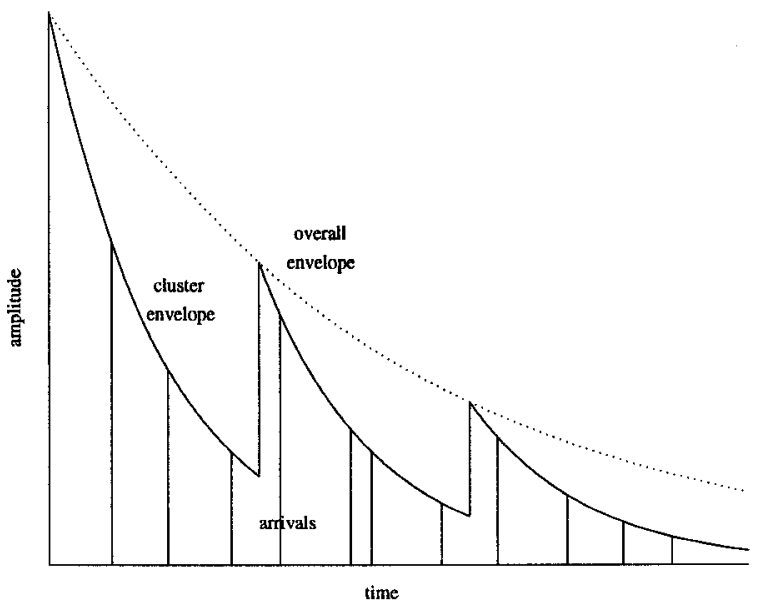

Fig. 1. An illustration of exponential decay of mean cluster power and ray power within clusters

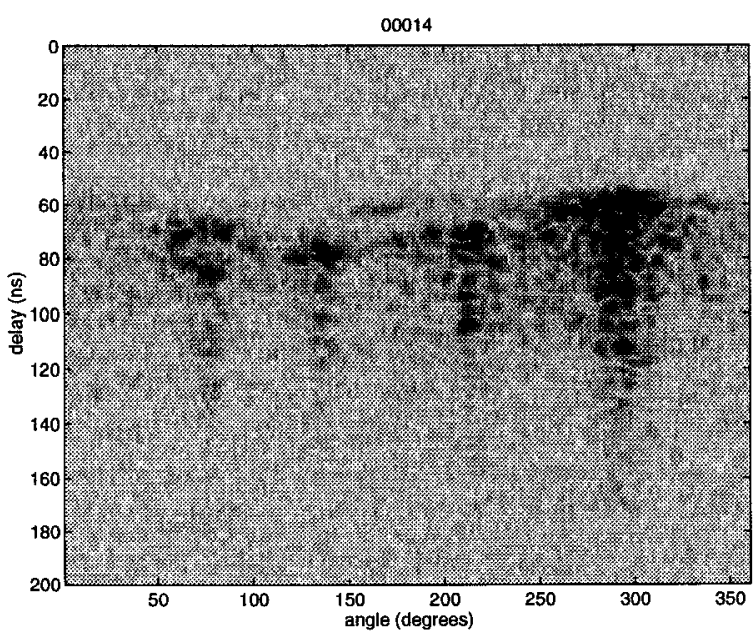

Fig. 2. A typical raw data set

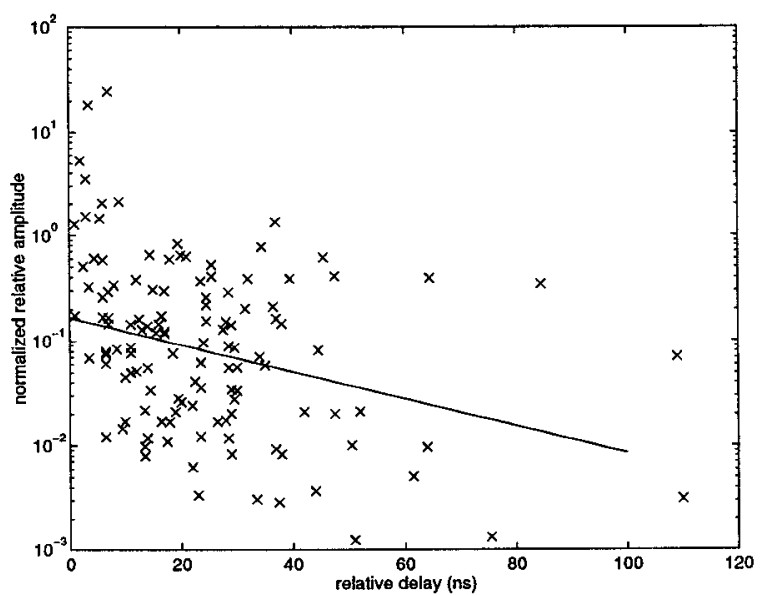

Fig. 3. Plot of normalized cluster amplitude vs. relative delay for the Clyde Building, with the curve for $\Gamma=33.6 \mathrm{~ns}$ superimposed.

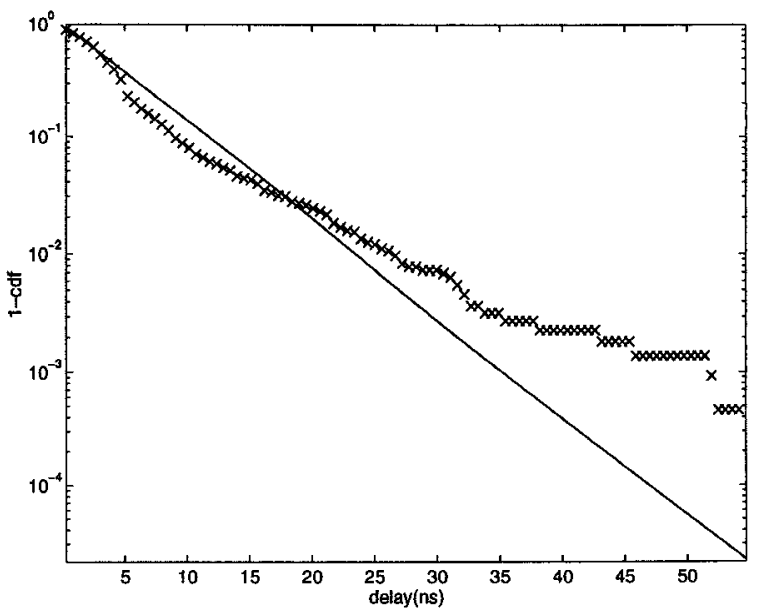

Fig. 4. CDF of Relative Arrival Times Within Clusters in the Clyde Building $(1 / \lambda=5.1 \mathrm{~ns})$

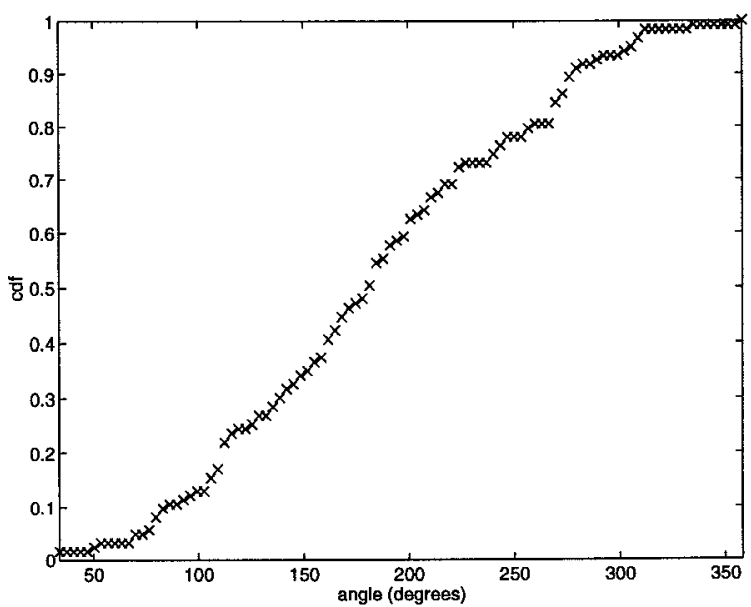

Fig. 5. CDF of relative mean cluster angles in the Clyde Building with respect to the first cluster in each set

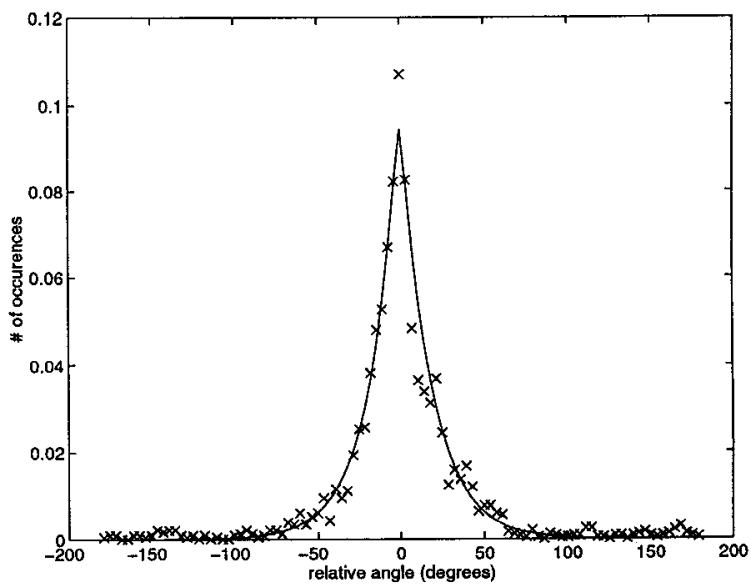

Fig. 6. Histogram of relative ray arrivals with respect to the cluster mean for the Clyde Building. Superimposed is the best fit Laplacian distribution $\left(\sigma=25.5^{\circ}\right)$. 